\title{
General formulae for dipole Wilson line correlators with the Color Glass Condensate
}

\author{
Kenji Fukushima $^{a}$ and Yoshimasa Hidaka ${ }^{b}$ \\ ${ }^{a}$ Department of Physics, The University of Tokyo, \\ 7-3-1 Hongo, Bunkyo-ku, Tokyo 113-0033, Japan \\ ${ }^{b}$ Theoretical Research Division, Nishina Center, RIKEN, \\ 2-1 Hirosawa, Wako, Saitama 351-0198, Japan \\ E-mail: fuku@nt.phys.s.u-tokyo.ac.jp, hidaka@riken.jp
}

ABSTRACT: We present general formulae to compute Wilson line correlators with the Color Glass Condensate described by the McLerran-Venugopalan model. We explicitly construct a complete and non-orthogonal set of color-singlet bases and write matrix elements down, so that the exponential of the matrix leads to the Wilson line correlators. We further develop a systematic perturbative expansion of dipole Wilson line correlators in terms of $1 / N_{\mathrm{c}}$ where $N_{\mathrm{c}}$ is the color number. As a phenomenological application we calculate the flow harmonics $v_{n}\{m\}$ in the dipole model and discuss the $N_{\mathrm{c}}$ scaling.

Keywords: Resummation, Perturbative QCD, Quark-Gluon Plasma

ArXiv EPrint: 1708.03051 


\section{Contents}

1 Introduction $\quad 1$

2 Master formulae $\quad 3$

3 Color singlet bases $\quad 4$

4 Dipole Wilson line correlators and the large- $N_{\mathrm{c}}$ expansion $\quad 7$

5 Comparison to the exact answer $\quad 10$

6 Flow harmonics and higher order contributions 11

$\begin{array}{lll}7 & \text { Conclusions } & 16\end{array}$

\section{Introduction}

Quantum Chromodynamics (QCD) has a special property called the asymptotic freedom that implies that the strong coupling constant runs to a smaller value in parton reactions involving harder momentum scales. In this way the perturbative calculation of QCD is a reliable theoretical description in high-energy nuclear physics. In reality, however, a naïve perturbative expansion breaks down for diffractive type reactions in which exchanged momenta are not necessary hard as compared to the collision energy. Then, it is indispensable to make a resummation over large logarithmic enhancement factors which appear kinematically. Such a resummation leads to a picture of increasing parton or gluon density with increasing scattering energy, and, one would anticipate that the parton density would eventually enter a new regime where the effect of parton overlapping is significant. In such a regime of dense partons, QCD is still perturbative in a sense that the running coupling constant $g$ is small, but is highly non-linear due to the gluon amplitude $A^{\mu}$ as large as $\sim 1 / g$ [and thus $g A^{\mu} \sim \mathcal{O}(1)$ ]. This non-linear dynamics is a manifestation of the parton or gluon saturation (see refs. [1-5] for pioneering extensions including the non-linearity, see also refs. [6, 7] for recent reviews).

The virtue of the gluon saturation is that physical observables exhibit universal behavior in terms of scaling variables, from which the saturation momentum, $Q_{\mathrm{s}}$, can be experimentally fixed [8]. The theoretical framework in the saturation regime to compute physical observables as functions of $Q_{\mathrm{s}}$ has been well established and known as the Color Glass Condensate (CGC) [9-11]. The CGC effective theory is elegantly formulated in the renormalization group language [12-14], in which the soft gluons are given by the classical Yang-Mills fields from the hard parton color source [15] whose transverse density is 
characterized by $Q_{\mathrm{s}}^{2}$. The non-linear quantum evolution equation for the color source distribution is known as the JIMWLK equation named after the authors of the pioneering works $[12-14,16,17]$. Although solving the JIMWLK equation demands huge computational resources (see, for example, ref. [18] for the reformulation using the Langevin equation, and refs. $[19,20]$ for numerical simulations), a Gaussian approximated solution has been derived [21, 22]. The simplest CGC model in the Gaussian approximation is commonly called the McLerran-Venugopalan (MV) model, which is often used as an initial input for the JIMWLK evolution, or, this model itself could provide us with a good description of the qualitative features of high-energy QCD processes.

One of the most interested and testable quantities calculated in the CGC framework is the particle production [23-26] and correlation [27-31], including electromagnetic probes [32-36]. In the MV model various correlations among gluons and quarks have been discussed, and these predictions are to be compared to the experimental data of jets and hadron correlations. One of the most well known examples is the CGC picture to understand the ridge structure seen in the di-jet or di-hadron correlations in rapidity space $[37,38]$. Thus, it would be a natural extension to apply the CGC-based calculations to account for the collective behavior in small size systems. As the collision energy grows up, small size systems such as the $p$ - $A$ (proton-nucleus) and even the $p-p$ collisions may look similar to the $A$ - $A$ collision, yielding systematic patterns of the flow observables (see ref. [39] for an experimental overview). The final state interaction might be still responsible for the flow observables, but at the same time, one must also estimate the initial state effect to quantify which of the initial and the final state interactions is more important. Recently, within the dipole model for the particle production the systematics of the higher order flow observables, $v_{n}\{m\}$ (i.e. $n$-th harmonics of the $m$-particle flow) has been quantified in the glasma graph approximation [40, 41] and in the full MV model [42, 43].

Generally, in the CGC model calculations, the most time-consuming part in numerics is the numerical computation of the expectation value of the Wilson lines. In the presence of the CGC background fields corresponding to the saturated soft gluons typically in the $p$ $A$ collision and the forward $p-p$ collision, one needs to take account of multiple scatterings which amount to the Wilson line in the eikonal approximation. It has been repeatedly discussed how to compute the Wilson line correlators efficiently in separate contexts (see ref. [44] for a very recent work toward general formulae for the Wilson line correlators, for instance). Therefore, it would be quite useful to establish a prescription to compute the Wilson line correlators with some generality but in a fairly straightforward way. There are several preceding works along these lines from an early attempt in ref. [45] to a very recent reformulation in ref. [46] on top of an explicit calculation in ref. [44]. The purpose of the present work is to translate the powerful method of ref. [45] to a more specific physics problem in a more handy form. In particular, the setup in ref. [45] was too general and it was not clear how to utilize the formulae for phenomenological applications. In the present work, hence, we focus on the special type of correlation function in terms of the dipole operators in the color fundamental representation, which is the basic building block in the dipole model [47]. For $n$-point dipole correlations the problem is reduced to the exponentiation of $n ! \times n !$ matrices. The calculation of the exponential of the matrix 
is numerically feasible but it is sometimes insightful to perform the large- $N_{\mathrm{c}}$ expansion, especially to identify the $N_{\mathrm{c}}$ scaling of observables. We emphasize that our formulae take a quite convenient form for the large- $N_{\mathrm{c}}$ expansion and we will demonstrate the expansion explicitly up to the order where the first nonzero flow cumulants appear. We check the validity of the large- $N_{\mathrm{c}}$ formulae by comparing to the exact results known for $n=2$ with $N_{\mathrm{c}}=3$. For $n \geq 4$ we need to be very careful of the correct large- $N_{\mathrm{c}}$ counting and we will argue the subtlety in the numerical analysis.

\section{Master formulae}

The Wilson line correlators that we calculate in this paper are expressed in analogy to quantum mechanics as follows [45]:

$$
\left\langle\prod_{i=1}^{n} U\left(\boldsymbol{x}_{\perp i}\right)_{\beta_{i} \alpha_{i}} U^{*}\left(\boldsymbol{y}_{\perp i}\right)_{\bar{\beta}_{i} \bar{\alpha}_{i}}\right\rangle=\exp \left[-\left(H_{0}+V\right)\right]_{\beta_{1} \bar{\beta}_{1} \cdots \beta_{n} \bar{\beta}_{n} ; \alpha_{1} \bar{\alpha}_{1} \cdots \alpha_{n} \bar{\alpha}_{n}}
$$

where the free Hamiltonian, $H_{0}$, is defined as

$$
H_{0}=Q_{\mathrm{s}}^{2} \frac{2 N_{\mathrm{c}}}{N_{\mathrm{c}}^{2}-1} L(0,0)\left[\sum_{i=1}^{n}\left(T_{\mathrm{F} i}^{a}-T_{\mathrm{F} i}^{* a}\right)\right]^{2} .
$$

Here, we take the summation over $a$ in group space (which is always implicitly assumed). In this paper we limit our considerations to the fundamental representation only and $T_{\mathrm{F} i}{ }^{a}$ 's represent the elements of $\mathrm{su}\left(N_{\mathrm{c}}\right)$ algebra in the fundamental representation, while the formula is valid for any representation. Here, $L(0,0)$ is quadratically divergent, so that only color states that have zero eigenvalues of $H_{0}$ can make finite contributions to the Wilson line correlators [and thus, the detailed definition of $L(0,0)$ is not important here; see ref. [45] for the explicit form of $L(0,0)$ ]. We note that the definition of $Q_{\mathrm{s}}$ is slightly different from refs. [42, 43] and we will come to this point later when we apply our formulae for the flow observables. For the moment, eq. (2.2) is understood as a definition of $Q_{\mathrm{s}}$ in our convention. The color matrix in the interaction part is

$$
\begin{aligned}
V=-Q_{\mathrm{s}}^{2} \frac{2 N_{\mathrm{c}}}{N_{\mathrm{c}}^{2}-1}\{ & \sum_{i>j}^{n}\left[T_{\mathrm{F} i}^{a} T_{\mathrm{F}}^{a} \Gamma\left(\left|\boldsymbol{x}_{\perp i}-\boldsymbol{x}_{\perp j}\right|\right)+T_{\mathrm{F} i}^{* a} T_{\mathrm{F}}^{* a} \Gamma\left(\left|\boldsymbol{y}_{\perp i}-\boldsymbol{y}_{\perp j}\right|\right)\right] \\
& \left.-\sum_{i, j=1}^{n} T_{\mathrm{F}}^{a} T_{\mathrm{F} j}^{* a} \Gamma\left(\left|\boldsymbol{x}_{\perp i}-\boldsymbol{y}_{\perp j}\right|\right)\right\}
\end{aligned}
$$

In the above the common building block, $\Gamma\left(\boldsymbol{x}_{\perp}\right)$, is defined in the CGC formalism by the following integral:

$$
\Gamma\left(\left|\boldsymbol{x}_{\perp}\right|\right):=2 g^{4} \int_{\Lambda}^{\infty} \frac{\mathrm{d} k}{2 \pi} \frac{1}{k^{3}}\left[1-J_{0}\left(k\left|\boldsymbol{x}_{\perp}\right|\right)\right] \simeq-\frac{g^{4}}{4 \pi}\left|\boldsymbol{x}_{\perp}\right|^{2} \ln \left(\left|\boldsymbol{x}_{\perp}\right| \bar{\Lambda}\right),
$$

where $J_{\alpha}(x)$ is the Bessel function of the first kind, $\Lambda$ is an infrared (IR) cutoff of order of $\Lambda_{\mathrm{QCD}}$ and we introduced a shorthand notation as $\bar{\Lambda}:=\frac{1}{2} \Lambda \mathrm{e}^{\gamma-1}$. This approximated 
expression has undesirable behavior in the IR region especially when $\left|\boldsymbol{x}_{\perp}\right| \bar{\Lambda}<1$. We cure this IR problem, according to ref. [43], by introducing another regulator as

$$
\Gamma\left(\left|\boldsymbol{x}_{\perp}\right|\right) \simeq \frac{g^{4}}{4 \pi}\left|\boldsymbol{x}_{\perp}\right|^{2} \ln \left(\frac{1}{\left|\boldsymbol{x}_{\perp}\right| \bar{\Lambda}}+\mathrm{e}\right) .
$$

We will adopt this regularized approximation for $\Gamma\left(\boldsymbol{x}_{\perp}\right)$ in our numerical calculations later in section 5 .

Now we shall sketch our strategy to proceed to concrete calculations of the Wilson line correlators using eq. (2.1). We will first identify all the color-singlet bases with which $H_{0}$ vanishes. For the $n$-th power product of $U$ and $U^{*}$, there are $2 n$ Wilson lines, and then the number of independent color-singlet bases should be $n$ !, as we will explain in the next section. We can easily construct the color index structures by taking the permutations. After confirming that $H_{0}$ surely vanishes with such bases, next, we will consider the matrix elements of $V$. In general it is not an easy task to find analytical expressions for the eigenvalues of $V$. Nevertheless, it is known that the large- $N_{\mathrm{c}}$ approximation works at good quantitative accuracy, and we will systematically make an expansion in power of $1 / N_{\mathrm{c}}$ to find analytical expressions.

\section{Color singlet bases}

Because of the dipole-type structures of the Wilson lines it is easy to find all the combinations of the color singlet indices. One trivial singlet is immediately found as

$$
\left\langle\{\alpha\} ;\{\bar{\alpha}\} \mid s_{0}\right\rangle:=N_{\mathrm{c}}^{-n / 2} \delta_{\alpha_{1} \bar{\alpha}_{1}} \cdots \delta_{\alpha_{n} \bar{\alpha}_{n}} .
$$

Clearly all the permutations are possible, i.e. we introduce $\left|s_{p}\right\rangle$ as a permutation of $\left|s_{0}\right\rangle$. For this purpose let us introduce the symmetry group $S_{n}$ with elements $\pi_{p}$ that denotes a permutation as

$$
\pi_{p}=\left(\begin{array}{cccc}
1 & 2 & \cdots & n \\
p_{1} & p_{2} & \cdots & p_{n}
\end{array}\right) .
$$

Then,

$$
\left\langle\{\alpha\} ;\{\bar{\alpha}\} \mid s_{p}\right\rangle:=\left\langle\left\{\pi_{p} \alpha\right\} ;\{\bar{\alpha}\} \mid s_{0}\right\rangle=N_{\mathrm{c}}^{-n / 2} \delta_{\alpha_{p_{1}} \bar{\alpha}_{1}} \cdots \delta_{\alpha_{p_{n}} \bar{\alpha}_{n}} .
$$

We note that any $\pi_{p}$ can be expressed as a product of cycles. For example, it is easy to confirm the following relation,

$$
\left(\begin{array}{llllll}
1 & 2 & 3 & 4 & 5 & 6 \\
5 & 1 & 3 & 6 & 2 & 4
\end{array}\right)=(152) \cdot(46),
$$

where one-cycles, i.e. (3) in the above case, are trivial and not explicitly written. Let us see several useful formulae for later calculation checks. Because of the well-known relation,

$$
2 T_{\mathrm{F} \beta_{i} \alpha_{i}}^{a} T_{\mathrm{F} \beta_{j} \alpha_{j}}^{a}=\delta_{\beta_{i} \alpha_{j}} \delta_{\beta_{j} \alpha_{i}}-\frac{1}{N_{\mathrm{c}}} \delta_{\beta_{i} \alpha_{i}} \delta_{\beta_{j} \alpha_{j}},
$$


we can easily prove that

$$
\left\langle\{\beta\} ;\{\bar{\beta}\}\left|2 T_{\mathrm{F} i}^{a} T_{\mathrm{F}}^{a}\right| s_{p}\right\rangle=\left\langle\left\{(i j) \pi_{p} \beta\right\} ;\{\bar{\beta}\} \mid s_{0}\right\rangle-\frac{1}{N_{\mathrm{c}}}\left\langle\left\{\pi_{p} \beta\right\} ;\{\bar{\beta}\} \mid s_{0}\right\rangle,
$$

which is schematically expressed as

$$
2 T_{\mathrm{F} i}^{a} T_{\mathrm{F}} \frac{a}{j} \cdot \pi_{p}=\left[(i j)-\frac{1}{N_{\mathrm{c}}}\right] \cdot \pi_{p} .
$$

For the complex conjugate, because $T_{\mathrm{F}}{ }^{a}$ 's are Hermitean, the following should hold:

$$
2 T_{\mathrm{F} \bar{\beta}_{i} \bar{\alpha}_{i}}^{* a} T_{\mathrm{F} \bar{\beta}_{j} \bar{\alpha}_{j}}^{* a}=\delta_{\bar{\alpha}_{j} \bar{\beta}_{i}} \delta_{\bar{\alpha}_{i} \bar{\beta}_{j}}-\frac{1}{N_{\mathrm{c}}} \delta_{\bar{\alpha}_{i} \bar{\beta}_{i}} \delta_{\bar{\alpha}_{j} \bar{\beta}_{j}},
$$

which implies

$$
\left\langle\{\beta\} ;\{\bar{\beta}\}\left|2 T_{\mathrm{F} i}^{* a} T_{\mathrm{F}}^{* a}\right| s_{p}\right\rangle=\left\langle\left\{\pi_{p} \beta\right\} ;\{(i j) \bar{\beta}\} \mid s_{0}\right\rangle-\frac{1}{N_{\mathrm{c}}}\left\langle\left\{\pi_{p} \beta\right\} ;\{\bar{\beta}\} \mid s_{0}\right\rangle .
$$

Thanks to the index structure of $\left|s_{0}\right\rangle$, we readily see; $\left\langle\left\{\pi_{p} \beta\right\} ;\{(i j) \bar{\beta}\} \mid s_{0}\right\rangle=$ $\left\langle\left\{\pi_{p}(i j)^{-1} \beta\right\} ;\{\bar{\beta}\} \mid s_{0}\right\rangle$ and trivially $(i j)^{-1}=(i j)$, so that we can again give a schematic representation as

$$
2 T_{\mathrm{F} i}^{* a} T_{\mathrm{F}}^{* a} \cdot \pi_{p}=\pi_{p} \cdot\left[(i j)-\frac{1}{N_{\mathrm{c}}}\right] .
$$

Also, another useful formula is

$$
2 T_{\mathrm{F} \beta_{i} \alpha_{i}}^{a} T_{\mathrm{F} \bar{\beta}_{j} \bar{\alpha}_{j}}^{* a}=\delta_{\beta_{i} \bar{\beta}_{j}} \delta_{\alpha_{i} \bar{\alpha}_{j}}-\frac{1}{N_{\mathrm{c}}} \delta_{\beta_{i} \alpha_{i}} \delta_{\bar{\beta}_{j} \bar{\alpha}_{j}} .
$$

In this case, if $p_{j}=i$, the contract of $\delta_{\beta_{i} \bar{\beta}_{j}} \delta_{\alpha_{i} \bar{\alpha}_{j}}=\delta_{\beta_{p_{j}} \bar{\beta}_{j}} \delta_{\alpha_{p_{j}} \bar{\alpha}_{j}}$ in the above and $\delta_{\alpha_{p_{j}} \bar{\alpha}_{j}}$ in $\left|s_{p}\right\rangle$ makes $N_{\mathrm{c}} \delta_{\beta_{p_{j}} \bar{\beta}_{j}}$. For $p_{j} \neq i$ the contract of $\delta_{\beta_{i} \bar{\beta}_{j}} \delta_{\alpha_{i} \bar{\alpha}_{j}}$ and $\delta_{\alpha_{p_{j}} \bar{\alpha}_{j}} \delta_{\alpha_{i} \bar{\alpha}_{p_{i}-1}}$ together with $\delta_{\beta_{p_{j}} \alpha_{p_{j}}} \delta_{\bar{\beta}_{p_{i}^{-1}} \bar{\alpha}_{p_{i}^{-1}}}$ (which is always implicitly taken for indices not involved in $T_{\mathrm{F}}{ }^{a}$ nor $\left.T_{\mathrm{F}}^{* a}\right)$ gives $\delta_{\beta_{i} \bar{\beta}_{j}} \delta_{\beta_{p_{j}} \bar{\beta}_{p_{i}^{-1}}}=\left(i p_{j}\right) \delta_{\beta_{p_{j}} \bar{\beta}_{j}} \delta_{\beta_{i} \bar{\beta}_{p_{i}^{-1}}}$, where $p_{i}^{-1}$ indicates an index that satisfies $p_{p_{i}^{-1}}=i$. Therefore, we establish the following schematic relations:

$$
2 T_{\mathrm{F} i}^{a} T_{\mathrm{F} j}^{* a} \pi_{p}= \begin{cases}\left(N_{\mathrm{c}}-\frac{1}{N_{\mathrm{c}}}\right) \cdot \pi_{p} & \text { for } p_{j}=i \\ {\left[\left(i p_{j}\right)-\frac{1}{N_{\mathrm{c}}}\right] \cdot \pi_{p}} & \text { for } p_{j} \neq i\end{cases}
$$

In what follows below, we will utilize the formulae (3.7), (3.10), and (3.12) to perform calculations with a compact notation.

It would be an instructive check to see how $H_{0}\left|s_{p}\right\rangle=0$ is satisfied for all $\left|s_{p}\right\rangle$. For this purpose we first need to expand the second-order Casimir operator in $H_{0}$ as

$$
\left[\sum_{i=1}^{n}\left(T_{\mathrm{F} i}^{a}-T_{\mathrm{F} i}^{* a}\right)\right]^{2}=\sum_{i=1}^{n}\left(T_{\mathrm{F} i}^{a 2}+T_{\mathrm{F} i}^{* a}\right)+2 \sum_{i>j}^{n}\left(T_{\mathrm{F} i}^{a} T_{\mathrm{F} j}^{a}+T_{\mathrm{F} i}^{* a} T_{\mathrm{F}}^{* a} \bar{j}\right)-2 \sum_{i, j=1}^{n} T_{\mathrm{F} i}^{a} T_{\mathrm{F}}^{* a} \dot{j} .
$$


Here, again, we note that the summation over $a$ is always implicitly assumed. The first term is nothing but the Casimir operator, so that it is simply given by

$$
\sum_{i=1}^{n}\left(T_{\mathrm{F} i}^{a 2}+T_{\mathrm{F} i}^{* a}\right)=n\left(N_{\mathrm{c}}-\frac{1}{N_{\mathrm{c}}}\right),
$$

according to the $\mathrm{su}\left(N_{\mathrm{c}}\right)$ algebra. Using the formulae (3.7), (3.10), and (3.12), we can simplify the rest (if applied to $\left|s_{p}\right\rangle$ ) as

$$
\begin{aligned}
& {\left[2 \sum_{i>j}^{n}\left(T_{\mathrm{F} i}^{a} T_{\mathrm{F} j}^{a}+T_{\mathrm{F}}^{*} \frac{a}{i} T_{\mathrm{F}}^{*} \frac{a}{j}\right)-2 \sum_{i, j=1}^{n} T_{\mathrm{F} i}^{a} T_{\mathrm{F}}^{*} \frac{a}{j}\right] \cdot \pi_{p}} \\
& =\sum_{i>j}^{n}\left\{\left[(i j)-\frac{1}{N_{\mathrm{c}}}\right] \cdot \pi_{p}+\pi_{p} \cdot\left[(i j)-\frac{1}{N_{\mathrm{c}}}\right]\right\}-\sum_{i, j=1}^{n}\left[N_{\mathrm{c}} \delta_{i p_{j}}+\left(i p_{j}\right)\left(1-\delta_{i p_{j}}\right)-\frac{1}{N_{\mathrm{c}}}\right] \cdot \pi_{p} \\
& =-n\left(N_{\mathrm{c}}-\frac{1}{N_{\mathrm{c}}}\right) \cdot \pi_{p}+\sum_{i>j}^{n}\left[(i j) \cdot \pi_{p}+\pi_{p} \cdot(i j)\right]-\sum_{i, j=1}^{n}\left(i p_{j}\right)\left(1-\delta_{i p_{j}}\right) \cdot \pi_{p} .
\end{aligned}
$$

The first term cancels out with eq. (3.14). Since $i$ and $j$ run from 1 to $n$, we can equivalently take a summation with respect to $j$ instead of using $p_{j}$, leading to

$$
-\sum_{i, j=1}^{n}\left(i p_{j}\right)\left(1-\delta_{i p_{j}}\right) \cdot \pi_{p}=-\sum_{i \neq j}^{n}(i j) \cdot \pi_{p} .
$$

Also, we see $\pi_{p} \cdot(i j)=\left(p_{i} p_{j}\right) \cdot \pi_{p}$, which allows us to replace $p_{i}$ and $p_{j}$ with $i$ and $j$ in the summations. Finally we arrive at

$$
\sum_{i>j}^{n}\left[(i j) \cdot \pi_{p}+\pi_{p} \cdot(i j)\right]-\sum_{i, j=1}^{n}\left(i p_{j}\right)\left(1-\delta_{i p_{j}}\right) \cdot \pi_{p}=0 .
$$

This completes our confirmation of $H_{0}\left|s_{p}\right\rangle=0$ for any $\left|s_{p}\right\rangle$.

For the practical calculation the most important is the evaluation of the matrix elements of $V$. In our formalism we can infer the matrix elements from

$$
\begin{gathered}
V \cdot \pi_{p}=-Q_{\mathrm{s}}^{2} \frac{N_{\mathrm{c}}}{N_{\mathrm{c}}^{2}-1}\left(\sum_{i>j}^{n}\left\{\left[(i j)-\frac{1}{N_{\mathrm{c}}}\right] \Gamma\left(\left|\boldsymbol{x}_{\perp i}-\boldsymbol{x}_{\perp j}\right|\right)+\left[\left(p_{i} p_{j}\right)-\frac{1}{N_{\mathrm{c}}}\right] \Gamma\left(\left|\boldsymbol{y}_{\perp i}-\boldsymbol{y}_{\perp j}\right|\right)\right\}\right. \\
\left.-\sum_{i, j=1}^{n}\left[N_{\mathrm{c}} \delta_{i p_{j}}+\left(i p_{j}\right)\left(1-\delta_{i p_{j}}\right)-\frac{1}{N_{\mathrm{c}}}\right] \Gamma\left(\left|\boldsymbol{x}_{\perp i}-\boldsymbol{y}_{\perp j}\right|\right)\right) \cdot \pi_{p}
\end{gathered}
$$

which can be easily verified with eqs. (3.7), (3.10), and (3.12). We can further simplify the above expression by introducing a notation for a proper combination of $\Gamma$ 's, i.e.,

$$
F\left(\boldsymbol{x}_{\perp}, \boldsymbol{x}_{\perp}^{\prime} ; \boldsymbol{y}_{\perp}, \boldsymbol{y}_{\perp}^{\prime}\right):=\Gamma\left(\left|\boldsymbol{x}_{\perp}^{\prime}-\boldsymbol{y}_{\perp}\right|\right)+\Gamma\left(\left|\boldsymbol{x}_{\perp}-\boldsymbol{y}_{\perp}^{\prime}\right|\right)-\Gamma\left(\left|\boldsymbol{x}_{\perp}-\boldsymbol{x}_{\perp}^{\prime}\right|\right)-\Gamma\left(\left|\boldsymbol{y}_{\perp}-\boldsymbol{y}_{\perp}^{\prime}\right|\right) .
$$

We then define the explicit components of the matrix elements as

$$
V\left|s_{p}\right\rangle=\sum_{p^{\prime}}\left|s_{p^{\prime}}\right\rangle V_{p^{\prime}, p}
$$


Here we must be careful of the fact that $\left|s_{p}\right\rangle$ 's span a complete set of bases but they are not orthogonal. Using these notations and definitions we can summarize the non-zero components as follows:

$$
\begin{aligned}
V_{p, p} & =Q_{\mathrm{s}}^{2}\left[\sum_{i=1}^{n} \Gamma\left(\left|\boldsymbol{x}_{\perp p_{i}}-\boldsymbol{y}_{\perp i}\right|\right)-\frac{1}{N_{\mathrm{c}}^{2}-1} \sum_{i>j}^{n} F\left(\boldsymbol{x}_{\perp p_{i}}, \boldsymbol{x}_{\perp p_{j}} ; \boldsymbol{y}_{\perp i}, \boldsymbol{y}_{\perp j}\right)\right], \\
V_{p(i j), p} & =Q_{\mathrm{s}}^{2} \frac{N_{\mathrm{c}}}{N_{\mathrm{c}}^{2}-1} F\left(\boldsymbol{x}_{\perp p_{i}}, \boldsymbol{x}_{\perp p_{j}} ; \boldsymbol{y}_{\perp i}, \boldsymbol{y}_{\perp j}\right),
\end{aligned}
$$

and other matrix elements vanish. These formulae are our central results in the present paper. For the actual application of the formulae, we should compute the exponential of $V$ as seen in eq. (2.1).

To understand how the formulae work, let us consider the simplest example of $n=2$. The matrix elements of $2 \times 2$ matrix $V$ read

$$
V=\left(\begin{array}{cc}
V_{0,0} & V_{0,(21)} \\
V_{(21), 0} & V_{(21),(21)}
\end{array}\right)=Q_{\mathrm{s}}^{2} \frac{2 N_{\mathrm{c}}}{N_{\mathrm{c}}^{2}-1}\left(\begin{array}{cc}
\frac{N_{\mathrm{c}}^{2}-1}{N_{\mathrm{c}}} \gamma-\frac{1}{N_{\mathrm{c}}}(\alpha-\beta) & \gamma-\beta \\
\alpha-\beta & \frac{N_{\mathrm{c}}^{2}-1}{N_{\mathrm{c}}} \alpha-\frac{1}{N_{\mathrm{c}}}(\gamma-\beta)
\end{array}\right),
$$

where,

$$
\begin{aligned}
& 2 \alpha:=\Gamma\left(\left|\boldsymbol{x}_{\perp 1}-\boldsymbol{y}_{\perp 2}\right|\right)+\Gamma\left(\left|\boldsymbol{y}_{\perp 1}-\boldsymbol{x}_{\perp 2}\right|\right), \\
& 2 \beta:=\Gamma\left(\left|\boldsymbol{x}_{\perp 1}-\boldsymbol{x}_{\perp 2}\right|\right)+\Gamma\left(\left|\boldsymbol{y}_{\perp 1}-\boldsymbol{y}_{\perp 2}\right|\right), \\
& 2 \gamma:=\Gamma\left(\left|\boldsymbol{x}_{\perp 1}-\boldsymbol{y}_{\perp 1}\right|\right)+\Gamma\left(\left|\boldsymbol{x}_{\perp 2}-\boldsymbol{x}_{\perp 2}\right|\right) .
\end{aligned}
$$

It is a straightforward calculation to obtain two eigenvalues as $\lambda_{ \pm}=\frac{1}{2}(\operatorname{tr} V \pm \varphi)$ with

$$
\varphi:=\sqrt{(\operatorname{tr} V)^{2}-4 \operatorname{det} V}=Q_{\mathrm{s}}^{2} \frac{2 N_{\mathrm{c}}^{2}}{N_{\mathrm{c}}^{2}-1} \sqrt{(\alpha-\gamma)^{2}+\frac{4}{N_{\mathrm{c}}^{2}}(\beta-\alpha)(\beta-\gamma)} .
$$

Now we can express the exponential of $V$ in a simple form as

$$
\mathrm{e}^{-V}=\mathrm{e}^{-\frac{1}{2} \operatorname{tr} V}\left(\begin{array}{cc}
\cosh \frac{1}{2} \varphi-\frac{\sinh \frac{1}{2} \varphi}{\varphi}\left(V_{0,0}-V_{(21),(21)}\right) & -2 \frac{\sinh \frac{1}{2} \varphi}{\varphi} V_{0,(21)} \\
-2 \frac{\sinh \frac{1}{2} \varphi}{\varphi} V_{(21), 0} & \cosh \frac{1}{2} \varphi+\frac{\sinh \frac{1}{2} \varphi}{\varphi}\left(V_{0,0}-V_{(21),(21)}\right)
\end{array}\right) .
$$

Although the calculation machinery is rather simple, a larger $n$ would cause a huge computational cost. Hence, we will seek for an algorithmic expansion to approximate $\mathrm{e}^{-V}$ without complicated matrix algebra.

\section{Dipole Wilson line correlators and the large- $N_{\mathrm{c}}$ expansion}

For the application for the particle production problem in the relativistic heavy-ion collision $[42,43]$, we are specifically interested in the correlation functions of the dipole operators. The definition of the dipole operator is

$$
D\left(\boldsymbol{x}_{\perp}, \boldsymbol{y}_{\perp}\right):=\frac{1}{N_{\mathrm{c}}} \operatorname{tr}\left[U\left(\boldsymbol{x}_{\perp}\right) U^{\dagger}\left(\boldsymbol{y}_{\perp}\right)\right]=\frac{1}{N_{\mathrm{c}}} \delta_{\beta \bar{\beta}} \delta_{\alpha \bar{\alpha}} U\left(\boldsymbol{x}_{\perp}\right)_{\beta \alpha} U^{*}\left(\boldsymbol{y}_{\perp}\right)_{\bar{\beta} \bar{\alpha}} .
$$


From this form it is obvious that the $n$ dipole expectation value is given by an matrix element of $\mathrm{e}^{-V}$ evaluated with $\left|s_{0}\right\rangle$, i.e.,

$$
\left\langle\prod_{i=1}^{n} D\left(\boldsymbol{x}_{\perp i}, \boldsymbol{y}_{\perp i}\right)\right\rangle=\left\langle s_{0}\left|\mathrm{e}^{-V}\right| s_{0}\right\rangle .
$$

We may be able to do a direct computation, but we can develop a more sophisticated method assuming that $N_{\mathrm{c}}$ is large enough. In view of eqs. (3.21) and (3.22), the offdiagonal components are suppressed by $1 / N_{\mathrm{c}}$, so that we can avoid exponentiating $V$ but make a systematic expansion in terms of $V_{p(i j), p}$.

For notational brevity we shall denote the diagonal and the off-diagonal parts of $V$ as $V^{(0)}$ and $V^{(1)}$, respectively. Then, the starting point for the systematic perturbative expansion is the interaction picture as in quantum mechanics expressed as

$$
\mathrm{e}^{-V}=\mathrm{e}^{-V^{(0)}} \mathcal{T}_{\tau} \exp \left[-\int_{0}^{1} \mathrm{~d} \tau V^{(1)}(\tau)\right]
$$

where $\mathcal{T}_{\tau}$ stands for the time-ordered product in terms of $\tau$ and the time dependent $V^{(1)}(\tau)$ in the interaction picture is defined as

$$
V^{(1)}(\tau):=\mathrm{e}^{\tau V^{(0)}} V^{(1)} \mathrm{e}^{-\tau V^{(0)}} .
$$

Thus, up to the second order in $V^{(1)}$ for example, the perturbative expansion reads

$$
\mathrm{e}^{-V} \simeq \mathrm{e}^{-V^{(0)}}\left[1-\int_{0}^{1} \mathrm{~d} \tau V^{(1)}(\tau)+\int_{0}^{1} \mathrm{~d} \tau_{1} \int_{0}^{\tau_{1}} \mathrm{~d} \tau_{2} V^{(1)}\left(\tau_{1}\right) V^{(1)}\left(\tau_{2}\right)\right]
$$

Because $V^{(0)}$ is a diagonal matrix, its matrix elements, $V_{p, p}$, are the eigenvalues of $V$. Then, let us introduce an eigenvector $|p\rangle$ with an eigenvalue $E_{p}$ for $V^{(0)}$. That is,

$$
V^{(0)}|p\rangle=E_{p}|p\rangle=\left(E_{p}^{(0)}+E_{p}^{(2)}\right)|p\rangle,
$$

where we decomposed the eigenvalue according to the $1 / N_{\mathrm{c}}$ order as

$$
\begin{aligned}
& E_{p}^{(0)}:=Q_{\mathrm{s}}^{2} \sum_{i=1}^{n} \Gamma\left(\boldsymbol{x}_{\perp p_{i}}-\boldsymbol{y}_{\perp i}\right) \\
& E_{p}^{(2)}:=-Q_{\mathrm{s}}^{2} \frac{1}{N_{\mathrm{c}}^{2}-1} \sum_{i>j}^{n} F\left(\boldsymbol{x}_{\perp p_{i}}, \boldsymbol{x}_{\perp p_{j}} ; \boldsymbol{y}_{\perp i}, \boldsymbol{y}_{\perp j}\right)=-\frac{1}{N_{\mathrm{c}}} \sum_{i>j}^{n} V_{p(i j), p}^{(1)} .
\end{aligned}
$$

It is then easy to re-express the first perturbative correction as

$$
\begin{aligned}
-\mathrm{e}^{-V^{(0)}} \int_{0}^{1} \mathrm{~d} \tau V^{(1)}(\tau)|p\rangle & =-\sum_{q}|q\rangle \mathrm{e}^{-E_{q}} \int_{0}^{1} \mathrm{~d} \tau V_{q p}^{(1)} \mathrm{e}^{\tau\left(E_{q}-E_{p}\right)} \\
& =\sum_{q}|q\rangle \frac{\mathrm{e}^{-E_{q}}-\mathrm{e}^{-E_{p}}}{E_{q}-E_{p}} V_{q p}^{(1)}
\end{aligned}
$$


Here, $V_{q p}^{(1)}$ is defined as $V^{(1)}|p\rangle=\sum_{q}|q\rangle V_{q p}^{(1)}$, where we note that this $V^{(1)}$ is an original matrix, not the one in the interaction picture.

In the same way, we can proceed to the second perturbative correction as

$$
\begin{aligned}
\mathrm{e}^{-V^{(0)}} & \int_{0}^{1} \mathrm{~d} \tau_{1} \int_{0}^{\tau_{1}} \mathrm{~d} \tau_{2} V^{(1)}\left(\tau_{1}\right) V^{(1)}\left(\tau_{2}\right)|p\rangle \\
& =\sum_{q, r}|q\rangle \mathrm{e}^{-E_{q}} \int_{0}^{1} \mathrm{~d} \tau_{1} \int_{0}^{\tau_{1}} \mathrm{~d} \tau_{2} \mathrm{e}^{\tau_{1}\left(E_{q}-E_{r}\right)+\tau_{2}\left(E_{r}-E_{p}\right)} V_{q r}^{(1)} V_{r p}^{(1)} \\
& =\sum_{q, r}|q\rangle\left(\frac{\mathrm{e}^{-E_{q}}-\mathrm{e}^{-E_{r}}}{E_{q}-E_{r}}-\frac{\mathrm{e}^{-E_{q}}-\mathrm{e}^{-E_{p}}}{E_{q}-E_{p}}\right) \frac{V_{q r}^{(1)} V_{r p}^{(1)}}{E_{r}-E_{p}}
\end{aligned}
$$

At this point, we can see a general algorithm to go to arbitrary high orders. The next order, for example, is generated automatically via one more iteration as

$$
\begin{aligned}
\sum_{q, s, r}|q\rangle & {\left[\left(\frac{\mathrm{e}^{-E_{q}}-\mathrm{e}^{-E_{s}}}{E_{q}-E_{s}}-\frac{\mathrm{e}^{-E_{q}}-\mathrm{e}^{-E_{r}}}{E_{q}-E_{r}}\right) \frac{1}{E_{s}-E_{r}}\right.} \\
& \left.-\left(\frac{\mathrm{e}^{-E_{q}}-\mathrm{e}^{-E_{s}}}{E_{q}-E_{s}}-\frac{\mathrm{e}^{-E_{q}}-\mathrm{e}^{-E_{p}}}{E_{q}-E_{p}}\right) \frac{1}{E_{s}-E_{p}}\right] \frac{V_{q s}^{(1)} V_{s r}^{(1)} V_{r p}^{(1)}}{E_{r}-E_{p}} .
\end{aligned}
$$

Now, we are ready to compute $\left\langle s_{0}\left|\mathrm{e}^{-V}\right| s_{0}\right\rangle$ up to the $N_{\mathrm{c}}^{-2}$ order. Noting that $V^{(1)}$ has a matrix element between $p$ and $p(i j)$, we can write down

$$
\begin{aligned}
\left\langle s_{0}\left|\mathrm{e}^{-V}\right| s_{0}\right\rangle \simeq\left\langle s_{0} \mid s_{0}\right\rangle \mathrm{e}^{-E_{0}} & +\sum_{i>j}^{n}\left\langle s_{0} \mid(i j) s_{0}\right\rangle \frac{\mathrm{e}^{-E_{(i j)}}-\mathrm{e}^{-E_{0}}}{E_{(i j)}-E_{0}} V_{(i j), 0}^{(1)} \\
& +\sum_{i>j}^{n}\left\langle s_{0} \mid s_{0}\right\rangle\left(\frac{\mathrm{e}^{-E_{0}}-\mathrm{e}^{-E_{(i j)}}}{E_{0}-E_{(i j)}}+e^{-E_{0}}\right) \frac{V_{0,(i j)}^{(1)} V_{(i j), 0}^{(1)}}{E_{(i j)}-E_{0}} .
\end{aligned}
$$

As we already mentioned, $\left|s_{p}\right\rangle$ 's are not orthogonal for different $p$ 's, and a simple calculation leads to the normalization as $\left\langle s_{0} \mid s_{p}\right\rangle=N_{\mathrm{c}}^{-n+n_{p}}$ where $n_{p}$ denotes the number of cycles of $\pi_{p}$. Because the second and the third terms are already suppressed by $N_{\mathrm{c}}^{-2}$, we can replace $E_{(i j)}$ with $E_{(i j)}^{(0)}$ in the above expansion. Then, we notice that $E_{(i j)}^{(0)}$ is always accompanied by $E_{0}^{(0)}$, which motivates us to introduce a new notation as

$\Delta E_{(i j)}^{(0)}:=E_{(i j)}^{(0)}-E_{0}^{(0)}=Q_{\mathrm{s}}^{2}\left[\Gamma\left(\left|\boldsymbol{x}_{\perp j}-\boldsymbol{y}_{\perp i}\right|\right)+\Gamma\left(\left|\boldsymbol{x}_{\perp i}-\boldsymbol{y}_{\perp j}\right|\right)-\Gamma\left(\left|\boldsymbol{x}_{\perp i}-\boldsymbol{y}_{\perp i}\right|\right)-\Gamma\left(\left|\boldsymbol{x}_{\perp j}-\boldsymbol{y}_{\perp j}\right|\right)\right]$.

Now, by expanding $E_{0}$ and using the above relations, we can reach the result from the large- $N_{\mathrm{c}}$ expansion up to the second order as

$$
\left\langle\prod_{i=1}^{n} D\left(\boldsymbol{x}_{\perp i}, \boldsymbol{y}_{\perp i}\right)\right\rangle=\mathrm{e}^{-E_{0}^{(0)}}\left\{1+\sum_{i>j}^{n}\left(1-\frac{1-\mathrm{e}^{-\Delta E_{(i j)}^{(0)}}}{\Delta E_{(i j)}^{(0)}}\right)\left(\frac{V_{(i j), 0}^{(1)}}{N_{\mathrm{c}}}+\frac{V_{0,(i j)}^{(1)} V_{(i j), 0}^{(1)}}{\Delta E_{(i j)}^{(0)}}\right)\right\} .
$$

This is our final expression expanded up to the $N_{\mathrm{c}}^{-2}$ order in the MV model. 


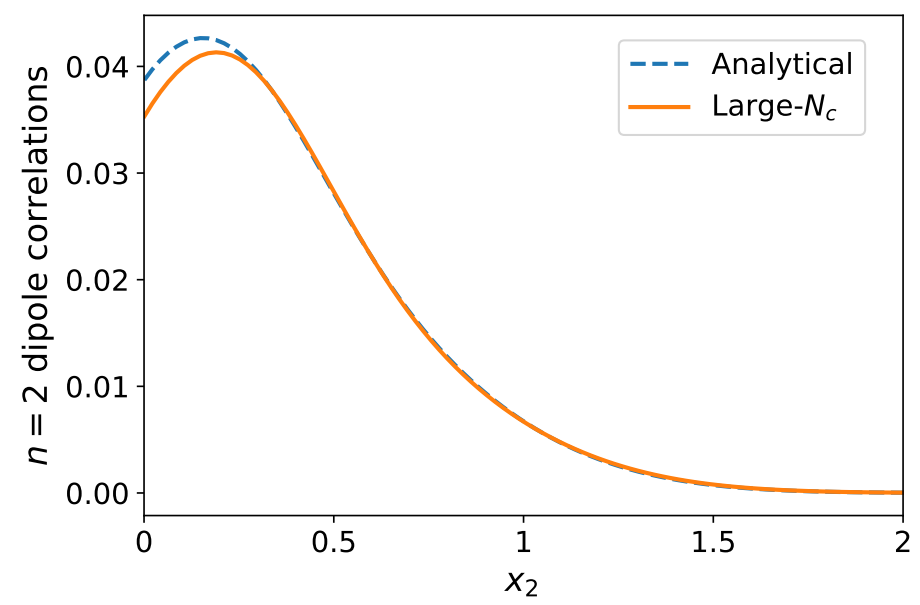

Figure 1. Validity check for the large- $N_{\mathrm{c}}$ approximation (4.14) as compared to the analytically exact result (3.26) for the $n=2$ dipole correlation with $Q_{\mathrm{s}}=5$ at $\boldsymbol{x}_{\perp 1}=(0,0), \boldsymbol{y}_{\perp 1}=(0.6,0.6)$, $\boldsymbol{x}_{\perp 2}=\left(x_{2}, 0\right), \boldsymbol{y}_{\perp 2}=(0.3,0.3)$ (in the unit of $\bar{\Lambda}$ ).

\section{Comparison to the exact answer}

In this section let us make a comparison between numerical results from our expansion (4.14) and the exact answer. In particular for the $n=2$ case as we discussed around eq. (3.26), the full analytical expression for the dipole Wilson line correlation is known for general $N_{\mathrm{c}}$, which provides us with a useful benchmark to quantify the validity of the large- $N_{\mathrm{c}}$ approximation in eq. (4.14). Because our present purpose is to check our formulae (4.14), concrete values of model parameters are not relevant. The coupling $g$ always appears as a combination of $g^{4} Q_{\mathrm{s}}^{2}$, so we can take $g=1$ without loss of generality and change $Q_{\mathrm{s}}$. We measure all variables in the unit of $\bar{\Lambda}$ here. This means, for example, $Q_{\mathrm{s}}=5$ in this section is actually $Q_{\mathrm{s}}=5 \bar{\Lambda}$, etc.

Figure 1 shows the validity check between eqs. (3.26) and (4.14) for the $n=2$ dipole correlator with $N_{\mathrm{c}}=3$. The agreement generally depends on $\boldsymbol{x}_{\perp i}$ and $\boldsymbol{y}_{\perp i}$, but our formulae (4.14) work quite well for almost all $\boldsymbol{x}_{\perp i}$ and $\boldsymbol{y}_{\perp i}$ as long as $Q_{\mathrm{s}}$ is not too large (when $Q_{\mathrm{s}}$ is too large, the outputs are too small, and the errors become relatively larger). Here, in figure 1 , we chose $Q_{\mathrm{s}}=5$ and $\boldsymbol{x}_{\perp 1}=(0,0), \boldsymbol{y}_{\perp 1}=(0.6,0.6), \boldsymbol{x}_{\perp 2}=\left(x_{2}, 0\right)$, $\boldsymbol{y}_{\perp 2}=(0.3,0.3)$, which is intentionally chosen to make the difference as visible as possible for the small $x_{2}$ region, and so, the overall agreement is better than shown in figure 1 .

We next see the $Q_{\mathrm{s}}$ dependence of the $n=2$ dipole correlator together with the Abelian approximation, as depicted in figure 2. We introduce the Abelian approximation as employed in refs. $[42,43]$ so that the $n=1$ expectation value can reproduce the exact result. For example of the $n=2$ case, the Abelian approximation reads

$$
\mathrm{e}^{-Q_{\mathrm{s}}^{2}\left[\Gamma\left(\left|\boldsymbol{x}_{\perp_{1}}-\boldsymbol{y}_{\perp_{1}}\right|\right)-\Gamma\left(\left|\boldsymbol{x}_{\perp_{1}}-\boldsymbol{x}_{\perp_{2}}\right|\right)+\Gamma\left(\left|\boldsymbol{x}_{\perp_{1}}-\boldsymbol{y}_{\perp_{2}}\right|\right)+\Gamma\left(\left|\boldsymbol{x}_{\perp_{2}}-\boldsymbol{y}_{\perp_{1}}\right|\right)-\Gamma\left(\left|\boldsymbol{y}_{\perp_{1}}-\boldsymbol{y}_{\perp_{2}}\right|\right)+\Gamma\left(\left|\boldsymbol{x}_{\perp_{2}}-\boldsymbol{y}_{\perp_{2}}\right|\right)\right]},
$$

which would agree with the exact answer in the limit of $\boldsymbol{x}_{\perp 1}=\boldsymbol{y}_{\perp 1}$ or $\boldsymbol{x}_{\perp 2}=\boldsymbol{y}_{\perp 2}$ (in which the correlator reduces to the $n=1$ one), but deviates from the exact answer for general $\boldsymbol{x}_{\perp i}$ and $\boldsymbol{y}_{\perp i}$. In this sense, the expression like eq. (5.1) is to be regarded as an Abelianized 


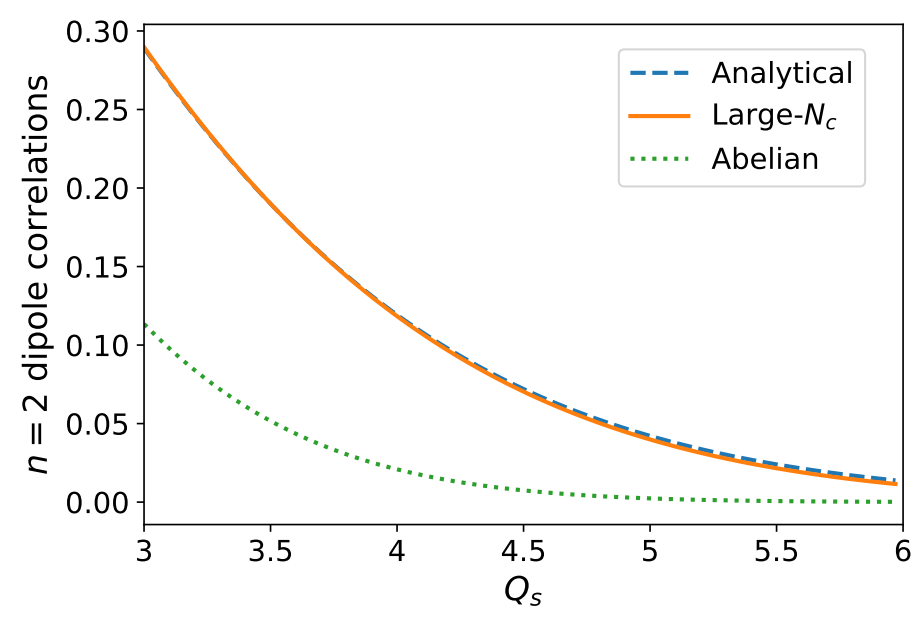

Figure 2. Validity check for the large- $N_{\mathrm{c}}$ approximation (4.14) and the Abelian approximation as compared to the analytically exact result (3.26) for the $n=2$ dipole correlation. The positions are chosen as $\boldsymbol{x}_{\perp 1}=(0,0), \boldsymbol{y}_{\perp 1}=(0.6,0.6), \boldsymbol{x}_{\perp 2}=(0.1,0), \boldsymbol{y}_{\perp 2}=(0.3,0.3)$.

extrapolation from the $n=1$ answer. Figure 2 clearly shows that a small disagreement magnified in figure 1 is actually a negligibly small difference in the whole profile over various $Q_{\mathrm{s}}$. The Abelian approximation captures a qualitative dependence with increasing $Q_{\mathrm{s}}$, while the quantitative values should be considered as only order estimates.

\section{Flow harmonics and higher order contributions}

We follow the calculations of the flow observables, $v_{n}\{m\}$, in the dilute-dense system according to refs. $[42,43]$, i.e. we focus on only the CGC effects on $v_{n}\{m\}$ apart from other fluctuations as implemented in the Monte-Carlo Glauber model. We here emphasize that our present goal is not giving a full theoretical prediction for the observables but quantifying the parton level contributions captured by the CGC model. The flows are characteristic angular distributions defined from the $m$-particle inclusive spectra, which are in the dipole model given by

$$
\begin{aligned}
& \frac{\mathrm{d}^{m} N}{\mathrm{~d}^{2} \boldsymbol{p}_{\perp 1} \cdots \mathrm{d}^{2} \boldsymbol{p}_{\perp m}} \\
& \quad=\frac{1}{\left(4 \pi^{3} B\right)^{m}} \prod_{i=1}^{m} \int \mathrm{d}^{2} \boldsymbol{x}_{\perp i} \mathrm{~d}^{2} \boldsymbol{y}_{\perp i} \mathrm{e}^{-\frac{\boldsymbol{x}_{\perp i}^{2}+\boldsymbol{y}_{\perp i}^{2}}{2 B}+\mathrm{i}\left(\boldsymbol{x}_{\perp i}-\boldsymbol{y}_{\perp i}\right) \cdot \boldsymbol{p}_{\perp i}}\left\langle\prod_{j=1}^{m} D\left(\boldsymbol{x}_{\perp j}, \boldsymbol{y}_{\perp j}\right)\right\rangle,
\end{aligned}
$$

where $B$ is a dipole model parameter. The dipole description is valid in the large $N_{\mathrm{c}}$ limit in which gluons are represented by dipoles. In principle, the dipole distribution should be fixed by the solution of the quantum evolution equation, but in the dipole model the distribution is simply approximated in a Gaussian form. Typically, the Gaussian size $B$ should be of order of the dilute-system (or proton) size $\sim 1 \mathrm{fm} \sim(0.2 \mathrm{GeV})^{-1}$, and we take $\sqrt{B}=2 \mathrm{GeV}^{-1}$.

We note that, if (multiple) gluon radiations occur, the above formula would be modified. Such processes (after the collision) are parametrically suppressed by higher powers of 
the strong coupling constant, but may be collinearly enhanced, leading to a parton shower. In the present analysis we implicitly assume that the initial parton correlations are not diminished by the hadronization process. The gluon splitting processes are also possible before the collision, but they should be identified as the parton evolution in the projectile wave-function, which is here approximated by the dipole model.

The general analysis for the flow properties is presented in ref. [48] and the $n$-th moment of the $m$-particle correlation is introduced as

$$
\kappa_{n}\{m\}:=\prod_{i=1}^{m} \int \frac{\mathrm{d}^{2} \boldsymbol{p}_{\perp i}}{(2 \pi)^{2}} \mathrm{e}^{\mathrm{i} n(-1)^{i+1} \phi_{i}} \frac{\mathrm{d}^{m} N}{\mathrm{~d}^{2} \boldsymbol{p}_{\perp 1} \cdots \boldsymbol{p}_{\perp m}},
$$

where $\phi_{i}$ represents the azimuthal angle, i.e. $\boldsymbol{p}_{\perp i}=\left|\boldsymbol{p}_{\perp i}\right|\left(\cos \phi_{i}, \sin \phi_{i}\right)$. Then, in the dipole model, we can perform the momentum integrations to find the following expression,

$\kappa_{n}\{m\}=\frac{1}{\left(4 \pi^{3} B\right)^{m}} \prod_{i=1}^{m} \int \mathrm{d}^{2} \boldsymbol{x}_{\perp i} \mathrm{~d}^{2} \boldsymbol{y}_{\perp i} \mathrm{e}^{-\frac{\boldsymbol{x}_{\perp i}^{2}+\boldsymbol{y}_{\perp i}^{2}}{2 B}} K_{n}^{\left((-1)^{i+1}\right)}\left(\boldsymbol{x}_{\perp i}-\boldsymbol{y}_{\perp i}\right)\left\langle\prod_{j=1}^{m} D\left(\boldsymbol{x}_{\perp j}, \boldsymbol{y}_{\perp j}\right)\right\rangle$.

Here, using the regularized generalized hypergeometric function, we defined,

$$
\begin{aligned}
K_{n}^{( \pm)}\left(\boldsymbol{x}_{\perp}\right) & :=\int^{p_{\max }} \frac{\mathrm{d}^{2} \boldsymbol{p}_{\perp}}{(2 \pi)^{2}} \mathrm{e}^{ \pm \mathrm{i} n \phi+\mathrm{i} \boldsymbol{x}_{\perp} \cdot \boldsymbol{p}_{\perp}} \\
& =\frac{\mathrm{i}^{n} p_{\max }^{2} \mathrm{e}^{ \pm \mathrm{i} n \theta}}{2 \pi(n+2) n !}\left(\frac{\left|\boldsymbol{x}_{\perp}\right| p_{\max }}{2}\right)^{n} F_{2}\left(1+\frac{n}{2} ;\left\{1+n, 2+\frac{n}{2}\right\} ;-\frac{1}{4}\left|\boldsymbol{x}_{\perp}\right|^{2} p_{\max }^{2}\right),
\end{aligned}
$$

where $\theta$ is the azimuthal angle of $\boldsymbol{x}_{\perp}$. In particular, we will frequently use the $n=0$ function for the normalization, which is given by

$$
K_{0}^{( \pm)}\left(\boldsymbol{x}_{\perp}\right)=\tilde{\delta}_{p_{\max }}^{(2)}\left(\boldsymbol{x}_{\perp}\right):=\frac{p_{\max }}{2 \pi\left|\boldsymbol{x}_{\perp}\right|} J_{1}\left(\left|\boldsymbol{x}_{\perp}\right| p_{\max }\right) .
$$

It is important to notice that \pm is irrelevant for $n=0$ and there is no angular dependence any more in $K_{0}^{( \pm)}\left(\boldsymbol{x}_{\perp}\right)$. Also, we must point out that $\tilde{\delta}_{p_{\max }}^{(2)}\left(\boldsymbol{x}_{\perp}\right)$ should approach $\delta^{(2)}\left(\boldsymbol{x}_{\perp}\right)$ in the $p_{\max } \rightarrow \infty$ limit.

Let us first consider the case with $m=2$ using our large- $N_{\mathrm{c}}$ expansion. The $N_{\mathrm{c}}^{0}$ order term in eq. (4.14) does not contribute to $\kappa_{n}\{2\}$ due to the phase factor in $K_{n}^{( \pm)}\left(\boldsymbol{x}_{\perp i}-\boldsymbol{y}_{\perp i}\right)$. As a result, we can write $\kappa_{n}\{2\}$ using the $N_{\mathrm{c}}^{-2}$ order results as

$$
\kappa_{n}\{2\}=D_{n}^{(+-)},
$$

where

$$
\begin{aligned}
D_{n}^{(+-)}:= & \frac{1}{\left(4 \pi^{3} B\right)^{2}} \int \mathrm{d}^{2} \boldsymbol{x}_{\perp 1} \mathrm{~d}^{2} \boldsymbol{y}_{\perp 1} \mathrm{~d}^{2} \boldsymbol{x}_{\perp 2} \mathrm{~d}^{2} \boldsymbol{y}_{\perp 2} \mathrm{e}^{-\frac{\boldsymbol{x}_{\perp 1}^{2}+\boldsymbol{y}_{\perp 1}^{2}+\boldsymbol{x}_{\perp 2}^{2}+\boldsymbol{y}_{\perp 2}^{2}}{2 B}} \\
& \times \mathrm{e}^{-Q_{\mathrm{s}}^{2} \Gamma\left(\boldsymbol{x}_{\perp 1}-\boldsymbol{y}_{\perp 1}\right)} K_{n}^{(+)}\left(\boldsymbol{x}_{\perp 1}-\boldsymbol{y}_{\perp 1}\right) \mathrm{e}^{-Q_{\mathrm{s}}^{2} \Gamma\left(\boldsymbol{x}_{\perp 2}-\boldsymbol{y}_{\perp 2}\right)} K_{n}^{(-)}\left(\boldsymbol{x}_{\perp 2}-\boldsymbol{y}_{\perp 2}\right) \\
& \times\left(1-\frac{1-\mathrm{e}^{-\Delta E_{(21)}^{(0)}}}{\Delta E_{(21)}^{(0)}}\right)\left(\frac{V_{(21), 0}^{(1)}}{N_{\mathrm{c}}}+\frac{V_{0,(21)}^{(1)} V_{(21), 0}^{(1)}}{\Delta E_{(21)}^{(0)}}\right)
\end{aligned}
$$




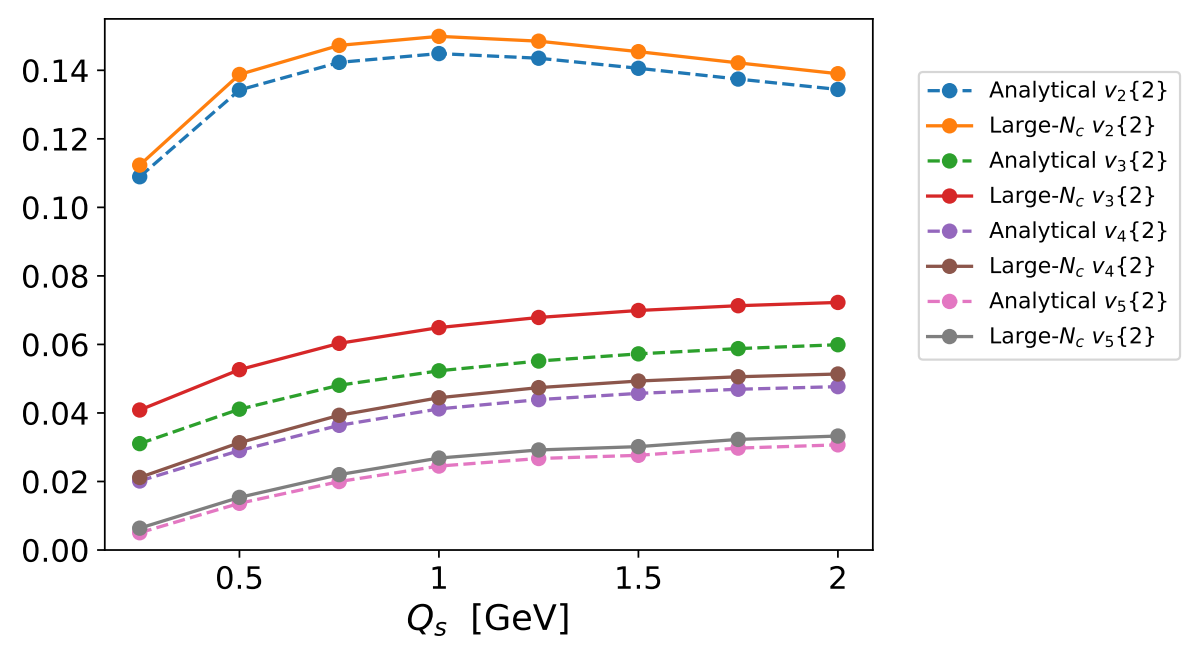

Figure 3. 2-particle flow harmonics using the analytical (unapproximated) expression (3.26) and our formulae (4.14). The saturation momentum $Q_{\mathrm{s}}$ is the one defined in eq. (6.11) as in ref. [43].

One could think of $D_{n}^{(++)}$and $D_{n}^{(--)}$in a similar manner but they are also vanishing because of the phase factors in $K_{n}^{( \pm)}$. The last part of the integrand is a function of modulus of various combinations of $\boldsymbol{x}_{\perp 1}, \boldsymbol{x}_{\perp 2}, \boldsymbol{y}_{\perp 1}, \boldsymbol{y}_{\perp 2}$. Here, it is crucially important to understand that any term in the integrand which is factorized into a function of $\left|\boldsymbol{x}_{\perp i}-\boldsymbol{y}_{\perp i}\right|$ alone would vanish due to the phase factors in $K_{n}^{( \pm)}\left(\boldsymbol{x}_{\perp_{i}}-\boldsymbol{y}_{\perp i}\right)$ in the factorized integrations.

Because there is no finite contribution of disconnected parts in the two particle correlation, we can immediately compute the two particle flow harmonics, $v_{n}\{2\}$, from

$$
v_{n}\{2\}=\sqrt{\frac{\kappa_{n}\{2\}}{\kappa_{0}\{2\}}},
$$

where the denominator is obtained with eq. (6.5), i.e. if we keep using the expanded expression up to the $N_{\mathrm{c}}^{-2}$ order for later convenience, we have

$$
\kappa_{0}\{2\}=D_{0}^{2}+D_{0}^{(+-)} .
$$

Here, we defined

$$
D_{0}:=\frac{1}{4 \pi^{3} B} \int \mathrm{d}^{2} \boldsymbol{x}_{\perp} \mathrm{d}^{2} \boldsymbol{y}_{\perp} \mathrm{e}^{-\frac{\boldsymbol{x}_{\perp}{ }^{2}+\boldsymbol{y}_{\perp}{ }^{2}}{2 B}} \mathrm{e}^{-Q_{\mathrm{s}}^{2} \Gamma\left(\boldsymbol{x}_{\perp}-\boldsymbol{y}_{\perp}\right)} \tilde{\delta}_{p_{\max }}^{(2)}\left(\boldsymbol{x}_{\perp}-\boldsymbol{y}_{\perp}\right) .
$$

Of course, up to this order, keeping $D_{0}^{(+-)}$in the denominator is in principle irrelevant since it gives a higher order correction which we should neglect.

We summarize our numerical results in figure 3 . We have performed the 8 dimensional numerical integration with respect to $\left\{\boldsymbol{x}_{\perp i}, \boldsymbol{y}_{\perp i}\right\}$ using the Monte-Carlo method by taking $10^{8}$ sampling points. To draw figure 3 we chose $\bar{\Lambda}=0.241 \mathrm{GeV}$ and $p_{\max }=2 \mathrm{GeV}$ in accord with ref. [43]. We also note that, only in this section, we change the definition of the saturation momentum from our original $Q_{\mathrm{s}}$ in eqs. (2.2) and (2.3) to new $\bar{Q}_{\mathrm{s}}$ defined by

$$
\bar{Q}_{\mathrm{s}}^{2}=\frac{1}{2 \Gamma\left(\left|\sqrt{2} / Q_{\mathrm{s}}\right|\right)}
$$


according to refs. $[42,43]$. Since there is no confusion, in this section, we will omit bar and simply denote $Q_{\mathrm{s}}$ to mean $\bar{Q}_{\mathrm{s}}$. Then, we can make a direct comparison of our outputs to figure 1 of ref. [42]. The dashed curved in figure 3 must precisely reproduce figure 1 of ref. [42]. At a glance of our numerical calculations we see quantitatively good agreement. The most interesting question is how useful our large- $N_{\mathrm{c}}$ formulae (4.14) can be for the $N_{\mathrm{c}}=3$ case. The comparison between the dashed curves (full analytical results) and the solid curves (large- $N_{\mathrm{c}}$ approximations) in figure 3 concludes that the errors are of only a few (at most $\sim 5$ ) \% level except for the $n=3$ case.

Next, it is intriguing to see what happens for $m=4$. In this case, $\kappa_{n}\{4\}$ involves $K_{n}^{(+)}\left(\boldsymbol{x}_{\perp 1}-\boldsymbol{y}_{\perp 1}\right), K_{n}^{(-)}\left(\boldsymbol{x}_{\perp 2}-\boldsymbol{y}_{\perp 2}\right), K_{n}^{(+)}\left(\boldsymbol{x}_{\perp 3}-\boldsymbol{y}_{\perp 3}\right)$, and $K_{n}^{(-)}\left(\boldsymbol{x}_{\perp 4}-\boldsymbol{y}_{\perp 4}\right)$. It is then easy to understand that our formula of the $N_{\mathrm{c}}^{-2}$ order in eq. (4.14) is insufficient to get nonvanishing contributions. Terms of the formula (4.14) are functions of, say, $\boldsymbol{x}_{\perp 1}, \boldsymbol{x}_{\perp 2}$, $\boldsymbol{y}_{\perp 1}, \boldsymbol{y}_{\perp 2}$ for $i=2$ and $j=1$, and then the angle integrations of $\boldsymbol{x}_{\perp 3}-\boldsymbol{y}_{\perp 3}$ and $\boldsymbol{x}_{\perp 4}-$ $\boldsymbol{y}_{\perp 4}$ become zero. Therefore, one permutation is not enough, but two permutations are necessary to shuffle all $\boldsymbol{x}_{\perp 1}, \boldsymbol{x}_{\perp 2}, \boldsymbol{x}_{\perp 3}$, and $\boldsymbol{x}_{\perp 4}$; we must go to the next $N_{\mathrm{c}}^{-4}$ order to compute a first nonzero term in $\kappa_{n}\{4\}$.

It is a straightforward but tedious calculation to pick all the $N_{\mathrm{c}}^{-4}$ order terms up from the expansion in eq. (4.11). We can slightly simplify the problem by discarding terms which do not contribute to $\kappa_{n}\{4\}$. The $N_{\mathrm{c}}^{-4}$ order terms generally contain the product of the interaction matrix elements like

$$
\sim V_{0,(p q)}^{(1)} V_{(p q),(i j)(k l)}^{(1)} V_{(i j)(k l),(i j)}^{(1)} V_{(i j), 0}^{(1)},
$$

but we already saw that, if an unexchanged pair exists, the angle integration is vanishing. For example, if the above matrix elements are $\sim V_{0,(21)}^{(1)} V_{(21), 0}^{(1)} V_{0,(21)}^{(1)} V_{(21), 0}^{(1)}$, which itself is nonzero, the angle integrations of $\boldsymbol{x}_{\perp 3}-\boldsymbol{y}_{\perp 3}$ and $\boldsymbol{x}_{\perp 4}-\boldsymbol{y}_{\perp 4}$ are zero. Thus, among all possible combinations of the matrix elements, there are finite contributions only from

$$
\begin{array}{ll}
V_{0,(43)}^{(1)} V_{(43),(0)}^{(1)} V_{(0),(21)}^{(1)} V_{(21), 0}^{(1)}, & V_{0,(42)}^{(1)} V_{(42),(0)}^{(1)} V_{(0),(31)}^{(1)} V_{(31), 0}^{(1)}, \\
V_{0,(32)}^{(1)} V_{(32),(0)}^{(1)} V_{(0),(41)}^{(1)} V_{(41), 0}^{(1)} . &
\end{array}
$$

Here, we used relations such as $V_{(i j)(k l),(i j)}^{(1)}=V_{(k l), 0}^{(1)}, V_{(k l),(i j)(k l)}^{(1)}=V_{0,(i j)}^{(1)}$, etc for $(i j) \neq(k l)$. After long calculations we arrive at a final form which turned out to be factorized as

$$
\begin{aligned}
& \kappa_{n}\{4\}= \frac{1}{\left(4 \pi^{3} B\right)^{4}} \prod_{i=1}^{4} \int \mathrm{d}^{2} \boldsymbol{x}_{\perp i} \mathrm{~d}^{2} \boldsymbol{y}_{\perp i} \mathrm{e}^{-\frac{\boldsymbol{x}_{\perp i}+\boldsymbol{y}_{\perp i}^{2}}{2 B}} \mathrm{e}^{-Q_{\mathrm{s}}^{2} \Gamma\left(\boldsymbol{x}_{\perp i}-\boldsymbol{y}_{\perp i}\right)} K_{n}^{\left((-1)^{i+1}\right)}\left(\boldsymbol{x}_{\perp i}-\boldsymbol{y}_{\perp i}\right) \\
& \times \frac{1}{2 !} \sum_{(i j) \neq(k l)}\left(1-\frac{1-\mathrm{e}^{-\Delta E_{(i j)}^{(0)}}}{\Delta E_{(i j)}^{(0)}}\right)\left(\frac{V_{(i j), 0}^{(1)}}{N_{\mathrm{c}}}+\frac{V_{0,(i j)}^{(1)} V_{(i j), 0}^{(1)}}{\Delta E_{(i j)}^{(0)}}\right) \\
& \times\left(1-\frac{1-\mathrm{e}^{-\Delta E_{(k l)}^{(0)}}}{\Delta E_{(k l)}^{(0)}}\right)\left(\frac{V_{(k l), 0}^{(1)}}{N_{\mathrm{c}}}+\frac{V_{0,(k l)}^{(1)} V_{(k l), 0}^{(1)}}{\Delta E_{(k l)}^{(0)}}\right)
\end{aligned}
$$

which is the full expression of the $N_{\mathrm{c}}^{-4}$ order without any truncation like the glasma graph approximation. This result looks quite reasonable, but we emphasize that the complete 
cancellation of $(i j)(k l)$ intermediate states with an energy denominator, $E_{(i j)}+E_{(k l)}$, is far from trivial. The sum with respect to $(i j)$ and $(k l)$ should run over all the permutations of the combinations as listed in eq. (6.13). Among all the combinations of indices, $[(i j)=$ $(31),(k l)=(42)]$ and $[(i j)=(42),(k l)=(31)]$ are irrelevant because $D_{n}^{(++)}=D_{n}^{(--)}=0$ (for $n>0$ ) as we already pointed out. Therefore, the remaining four combinations of $[(i j)=(21),(k l)=(43)],[(i j)=(43),(k l)=(21)],[(i j)=(41),(k l)=(32)]$, and $[(i j)=$ $(32),(k l)=(41)]$ lead to

$$
\kappa_{n}\{4\}=2\left[D_{n}^{(+-)}\right]^{2} .
$$

Then, with extra terms corresponding to $D_{0}^{(++)}$and $D_{0}^{(--)}$which are nonzero, the normalization is written as

$$
\kappa_{0}\{4\}=D_{0}^{4}+6 D_{0}^{2} D_{0}^{(+-)},
$$

up to the $N_{\mathrm{c}}^{-2}$ order in the same way as in eq. (6.9). Now, the cumulant is then given by

$$
c_{n}\{4\}=\frac{\kappa_{n}\{4\}}{\kappa_{0}\{4\}}-2\left(\frac{\kappa_{n}\{2\}}{\kappa_{0}\{2\}}\right)^{2} .
$$

The above quantity itself is zero in the strict order counting for $c_{n}\{4\}$ up to $N_{\mathrm{c}}^{-4}$. Thus, our conclusion is, even in the full MV model beyond the glasma graph approximation, no connected cumulant remains for $c_{n}\{4\}$ at the $N_{\mathrm{c}}^{-4}$ order.

In this way we can understand that the first connected contribution to cumulants appears from the $N_{\mathrm{c}}^{-2 m+2}$ order; for $m=4$, thus, we need to go to the $N_{\mathrm{c}}^{-6}$ order and then a completely nested combination of four indices like $(43),(32),(21)$ is possible. Therefore, the flow harmonics from the fully nested permutations must scale as

$$
v_{n}\{m\} \sim N_{\mathrm{c}}^{-2+2 / m} .
$$

This is our conclusion on the $N_{\mathrm{c}}$ scaling in the full MV model. We make a remark that this conclusion is robust even if the MV model is extended to include non-Gaussian (cubic, quartic, etc) terms. For example, one insertion of quartic vertex would decrease one $1 / N_{\mathrm{c}}$ factor to make a completely nested diagram, but one extra $g^{2} \sim N_{\mathrm{c}}$ factor from the quartic coupling would compensate for it to give the same scaling after all.

Although our conclusion of $c_{n}\{4\}=0$ at the $N_{\mathrm{c}}^{-4}$ order is solid, there may be a subtle point in the large- $N_{\mathrm{c}}$ scaling due to finiteness of $p_{\max }$. If one just uses our formulae up to the $N_{\mathrm{c}}^{-4}$ order to estimate $\kappa_{n}\{2\}, \kappa_{0}\{2\}, \kappa_{n}\{4\}$, and $\kappa_{0}\{4\}$, one would naïvely find that the cancellation is incomplete at the $N_{\mathrm{c}}^{-6}$ order and a finite remainder is given by

$\delta c_{n}\{4\}=\frac{2\left[D_{n}^{(+-)}\right]^{2}}{D_{0}^{4}+6 D_{0}^{2} D_{0}^{(+-)}}-2\left(\frac{D_{n}^{(+-)}}{D_{0}^{2}+D_{0}^{(+-)}}\right)^{2} \simeq-\frac{8\left[D_{n}^{(+-)}\right]^{2} D_{0}^{(+-)}}{D_{0}^{6}} \simeq-\frac{8 D_{0}^{(+-)}}{D_{0}^{2}}\left(v_{n}\{2\}\right)^{4}$.

This correction is beyond the $N_{\mathrm{c}}^{-4}$ order and should be identified as a part of the $N_{\mathrm{c}}^{-6}$ terms. One can easily check that this $N_{\mathrm{c}}^{-6}$ order correction is vanishing for $p_{\max } \rightarrow \infty$, in which $D_{0}^{(+-)} \rightarrow 0$. However, for a finite $p_{\max }$ especially comparable to $Q_{\mathrm{s}}$, the correction could be sizable and the flow harmonics is modified even from the disconnected piece. To 


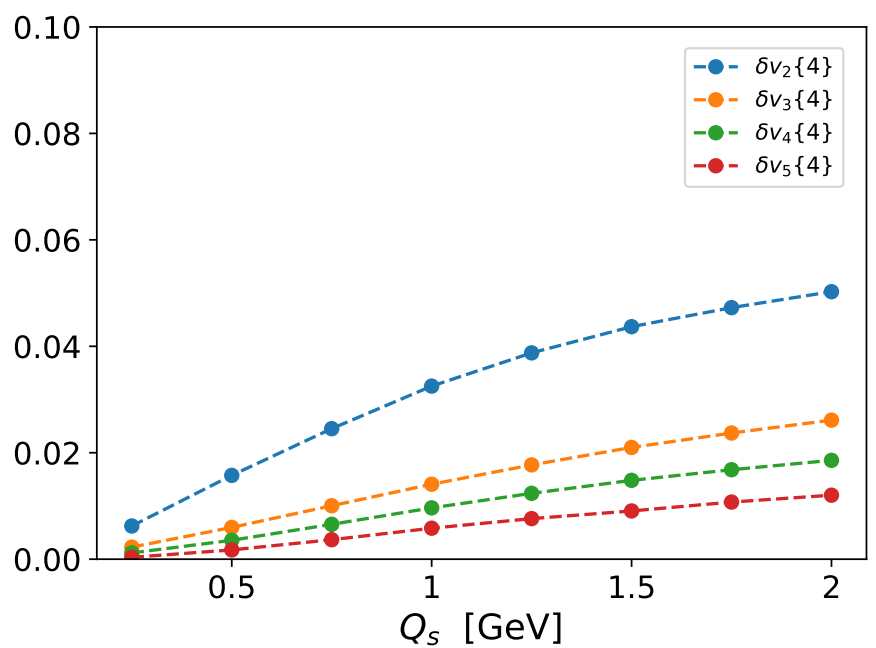

Figure 4. $\delta v_{n}\{4\}$ estimated from eq. (6.20) using $v_{n}\{2\}$ obtained in the large- $N_{\mathrm{c}}$ limit. Our choice of the parameter is $p_{\max }=2 \mathrm{GeV}$.

see this effect quantitatively, let us compute the flow harmonics corresponding to $\delta c\{4\}$, which leads to

$$
\delta v_{n}\{4\}=\left(-\delta c_{n}\{4\}\right)^{1 / 4}=2^{3 / 4}\left(\frac{D_{0}^{(+-)}}{D_{0}^{2}}\right)^{1 / 4} v_{n}\{2\} .
$$

We make a plot of $\delta v_{n}\{4\}$ as a function of $Q_{\mathrm{s}}$ in figure 4 . One must be very careful of the physical interpretation of this correction by $\delta v_{n}\{4\}$. Even though this is non-negligible as seen in figure 4, the physical origin lies in not the connected correlator but in the normalization. Moreover, this normalization effect makes the $N_{\mathrm{c}}$ counting skewed to become

$$
\delta v_{n}\{m\} \sim N_{\mathrm{c}}^{-1-2 / m} \quad(m \geq 4),
$$

which starts differing from anticipated eq. (6.18) for $m>4$. Thus, to distinguish the connected contribution from the normalization effect, one can test the $N_{\mathrm{c}}$ scaling properties as in eqs. (6.18) and (6.21) and also check the $p_{\max }$ dependence since $\delta v_{n}\{m\}$ from the normalization effect is very sensitive to $p_{\max } / Q_{\mathrm{s}}$ as perceived from figure 4 .

\section{Conclusions}

We have established general formulae and machineries to compute $2 n$-point Wilson line (or $n$ dipole) correlators with the color group representation $\left(N_{\mathrm{c}} \otimes \bar{N}_{\mathrm{c}}\right)^{n}$ in the McLerranVenugopalan model. The color structure accommodates a huge representation but the nonzero contribution to the Wilson line correlators reduces to the $n ! \times n !$ matrix, whose bases correspond to the color singlets. In particular, we have derived the explicit expression of the matrix elements [see eqs. (3.21) and (3.22)] in the color singlet bases constructed by permutations. The formulae are quite powerful not only for the direct numerical evaluation of the matrix but also for the analytical large- $N_{\mathrm{c}}$ expansion. We have developed the systematic large- $N_{\mathrm{c}}$ expansion in a way analogous to time-dependent perturbation theory 
in quantum mechanics. We have then shown the explicit expression up to $N_{\mathrm{c}}^{-2}$ order for the dipole correlators as given in eq. (4.14). As a check of the validity, we have compared results from the exact answers and those in the large- $N_{\mathrm{c}}$ expansion for the two-particle flow harmonics, $v_{n}\{2\}(n=2,3,4,5)$, which shows quantitatively good agreement. We have continued our large- $N_{\mathrm{c}}$ expansion to higher orders to discuss the flow harmonics with more particles. Then, we have discovered the $N_{\mathrm{c}}$ scaling as $v_{n}\{m\} \sim N_{\mathrm{c}}^{-2+2 / m}$ even beyond the glasma graph approximation but in the full MV model. We have also pointed out that a slightly different $N_{\mathrm{c}}$ scaling could emerge from the normalization effect at finite cutoff of the transverse momenta of integrated particles.

Although we focused on only the dipole correlators in the present paper, our general formulae also provide us with useful approaches to evaluate Wilson line correlators in channels relevant for the particle production rate in the $p$ - $A$ collision generally. In fact, not only fundamental but also adjoint Wilson line correlators appearing in the multi-gluon production can be derived from our results in eq. (2.1) through the relation $\left[U_{\text {adj }}\right]_{b a}\left(\boldsymbol{x}_{\perp}\right)=$ $2 \operatorname{tr}\left[U\left(\boldsymbol{x}_{\perp}\right) T_{\mathrm{F}}{ }^{a} U^{\dagger}\left(\boldsymbol{x}_{\perp}\right) T_{\mathrm{F}}^{b}\right]=T_{\mathrm{F}}^{a} \alpha \bar{\alpha} T_{\mathrm{F}}{ }_{\bar{\beta} \beta}^{b} U_{\beta \alpha}\left(\boldsymbol{x}_{\perp}\right) U_{\bar{\beta} \bar{\alpha}}^{*}\left(\boldsymbol{x}_{\perp}\right)$.

As we emphasized, our scheme of the large- $N_{\mathrm{c}}$ expansion takes a nice form which is easily implemented in numerical algorithms to go to arbitrarily higher orders. Such higher order numerical evaluations are left as an intriguing future problem. It would be also an important question to think about generalizations beyond the MV model. Further systematic considerations on the Wilson line correlators should deserve more investigations in the future.

\section{Acknowledgments}

The authors thank Kevin Dusling, Mark Mace, Sören Schlichting, Vladimir Skokov, and Raju Venugopalan for discussions. This work was supported by Japan Society for the Promotion of Science (JSPS) KAKENHI Grant No. 15H03652, 15 K13479 and 16K17716.

Open Access. This article is distributed under the terms of the Creative Commons Attribution License (CC-BY 4.0), which permits any use, distribution and reproduction in any medium, provided the original author(s) and source are credited.

\section{References}

[1] A.H. Mueller, Soft gluons in the infinite momentum wave function and the BFKL Pomeron, Nucl. Phys. B 415 (1994) 373 [InSPIRE].

[2] A.H. Mueller and B. Patel, Single and double BFKL Pomeron exchange and a dipole picture of high-energy hard processes, Nucl. Phys. B 425 (1994) 471 [hep-ph/9403256] [INSPIRE].

[3] I. Balitsky, Operator expansion for high-energy scattering, Nucl. Phys. B 463 (1996) 99 [hep-ph/9509348] [INSPIRE].

[4] I. Balitsky, Effective field theory for the small x evolution, Phys. Lett. B 518 (2001) 235 [hep-ph/0105334] [INSPIRE].

[5] Y.V. Kovchegov, Small $x F_{2}$ structure function of a nucleus including multiple Pomeron exchanges, Phys. Rev. D 60 (1999) 034008 [hep-ph/9901281] [INSPIRE]. 
[6] F. Gelis, Color glass condensate and glasma, Int. J. Mod. Phys. A 28 (2013) 1330001 [arXiv:1211.3327] [INSPIRE].

[7] J.-P. Blaizot, High gluon densities in heavy ion collisions, Rept. Prog. Phys. 80 (2017) 032301 [arXiv: 1607.04448] [INSPIRE].

[8] A.M. Stasto, K.J. Golec-Biernat and J. Kwiecinski, Geometric scaling for the total $\gamma^{*} p$ cross-section in the low $x$ region, Phys. Rev. Lett. 86 (2001) 596 [hep-ph/0007192] [INSPIRE].

[9] L.D. McLerran and R. Venugopalan, Computing quark and gluon distribution functions for very large nuclei, Phys. Rev. D 49 (1994) 2233 [hep-ph/9309289] [InSPIRE].

[10] L.D. McLerran and R. Venugopalan, Gluon distribution functions for very large nuclei at small transverse momentum, Phys. Rev. D 49 (1994) 3352 [hep-ph/9311205] [INSPIRE].

[11] L.D. McLerran and R. Venugopalan, Green's functions in the color field of a large nucleus, Phys. Rev. D 50 (1994) 2225 [hep-ph/9402335] [INSPIRE].

[12] J. Jalilian-Marian, A. Kovner, A. Leonidov and $\mathrm{H}$. Weigert, The BFKL equation from the Wilson renormalization group, Nucl. Phys. B 504 (1997) 415 [hep-ph/9701284] [INSPIRE].

[13] J. Jalilian-Marian, A. Kovner, A. Leonidov and H. Weigert, The Wilson renormalization group for low $x$ physics: towards the high density regime, Phys. Rev. D 59 (1998) 014014 [hep-ph/9706377] [INSPIRE].

[14] J. Jalilian-Marian, A. Kovner and H. Weigert, The Wilson renormalization group for low $x$ physics: gluon evolution at finite parton density, Phys. Rev. D 59 (1998) 014015 [hep-ph/9709432] [INSPIRE].

[15] Y.V. Kovchegov, Non-Abelian Weizsacker-Williams field and a two-dimensional effective color charge density for a very large nucleus, Phys. Rev. D 54 (1996) 5463 [hep-ph/9605446] [INSPIRE].

[16] E. Iancu, A. Leonidov and L.D. McLerran, Nonlinear gluon evolution in the color glass condensate. 1, Nucl. Phys. A 692 (2001) 583 [hep-ph/0011241] [INSPIRE].

[17] E. Ferreiro, E. Iancu, A. Leonidov and L. McLerran, Nonlinear gluon evolution in the color glass condensate. 2, Nucl. Phys. A 703 (2002) 489 [hep-ph/0109115] [INSPIRE].

[18] H. Weigert, Unitarity at small Bjorken x, Nucl. Phys. A 703 (2002) 823 [hep-ph/0004044] [INSPIRE].

[19] K. Rummukainen and H. Weigert, Universal features of JIMWLK and BK evolution at small x, Nucl. Phys. A 739 (2004) 183 [hep-ph/0309306] [INSPIRE].

[20] T. Lappi and H. Mäntysaari, Direct numerical solution of the coordinate space Balitsky-Kovchegov equation at next to leading order, Phys. Rev. D 91 (2015) 074016 [arXiv: 1502.02400] [INSPIRE].

[21] E. Iancu, K. Itakura and L. McLerran, A Gaussian effective theory for gluon saturation, Nucl. Phys. A 724 (2003) 181 [hep-ph/0212123] [INSPIRE].

[22] T. Lappi and H. Mantysaari, Forward dihadron correlations in deuteron-gold collisions with the Gaussian approximation of JIMWLK, Nucl. Phys. A 908 (2013) 51 [arXiv:1209.2853] [INSPIRE].

[23] A. Kovner and U.A. Wiedemann, Eikonal evolution and gluon radiation, Phys. Rev. D 64 (2001) 114002 [hep-ph/0106240] [INSPIRE]. 
[24] Y.V. Kovchegov and K. Tuchin, Inclusive gluon production in DIS at high parton density, Phys. Rev. D 65 (2002) 074026 [hep-ph/0111362] [InSPIRE].

[25] J.P. Blaizot, F. Gelis and R. Venugopalan, High-energy $p A$ collisions in the color glass condensate approach. 1. Gluon production and the Cronin effect, Nucl. Phys. A 743 (2004) 13 [hep-ph/0402256] [INSPIRE].

[26] J.P. Blaizot, F. Gelis and R. Venugopalan, High-energy $p A$ collisions in the color glass condensate approach. 2. Quark production, Nucl. Phys. A 743 (2004) 57 [hep-ph/0402257] [INSPIRE].

[27] R. Baier, A. Kovner, M. Nardi and U.A. Wiedemann, Particle correlations in saturated QCD matter, Phys. Rev. D 72 (2005) 094013 [hep-ph/0506126] [INSPIRE].

[28] C. Marquet, Forward inclusive dijet production and azimuthal correlations in $p A$ collisions, Nucl. Phys. A 796 (2007) 41 [arXiv:0708.0231] [INSPIRE].

[29] K. Fukushima and Y. Hidaka, Two gluon production and longitudinal correlations in the color glass condensate, Nucl. Phys. A 813 (2008) 171 [arXiv:0806.2143] [InSPIRE].

[30] J.L. Albacete and C. Marquet, Azimuthal correlations of forward di-hadrons in $d+A u$ collisions at RHIC in the color glass condensate, Phys. Rev. Lett. 105 (2010) 162301 [arXiv: 1005.4065] [INSPIRE].

[31] A. Kovner, M. Lublinsky and V. Skokov, Exploring correlations in the CGC wave function: odd azimuthal anisotropy, Phys. Rev. D 96 (2017) 016010 [arXiv:1612.07790] [INSPIRE].

[32] F. Gelis and J. Jalilian-Marian, Photon production in high-energy proton nucleus collisions, Phys. Rev. D 66 (2002) 014021 [hep-ph/0205037] [INSPIRE].

[33] F. Gelis and J. Jalilian-Marian, Dilepton production from the color glass condensate, Phys. Rev. D 66 (2002) 094014 [hep-ph/0208141] [INSPIRE].

[34] J. Jalilian-Marian, Electromagnetic signatures of the color glass condensate: dileptons, Nucl. Phys. A 739 (2004) 319 [nucl-th/0402014] [INSPIRE].

[35] S. Benic and K. Fukushima, Photon from the annihilation process with $C G C$ in the $p A$ collision, Nucl. Phys. A 958 (2017) 1 [arXiv:1602.01989] [INSPIRE].

[36] S. Benic, K. Fukushima, O. Garcia-Montero and R. Venugopalan, Probing gluon saturation with next-to-leading order photon production at central rapidities in proton-nucleus collisions, JHEP 01 (2017) 115 [arXiv: 1609.09424] [INSPIRE].

[37] A. Dumitru, F. Gelis, L. McLerran and R. Venugopalan, Glasma flux tubes and the near side ridge phenomenon at RHIC, Nucl. Phys. A 810 (2008) 91 [arXiv:0804.3858] [InSPIRE].

[38] A. Dumitru, K. Dusling, F. Gelis, J. Jalilian-Marian, T. Lappi and R. Venugopalan, The ridge in proton-proton collisions at the LHC, Phys. Lett. B 697 (2011) 21 [arXiv: 1009.5295] [INSPIRE].

[39] C. Loizides, Experimental overview on small collision systems at the LHC, Nucl. Phys. A 956 (2016) 200 [arXiv: 1602.09138] [INSPIRE].

[40] V. Skokov, High order cumulants of the azimuthal anisotropy in the dilute-dense limit: connected graphs, Phys. Rev. D 91 (2015) 054014 [arXiv: 1412.5191] [INSPIRE].

[41] T. Lappi, B. Schenke, S. Schlichting and R. Venugopalan, Tracing the origin of azimuthal gluon correlations in the color glass condensate, JHEP 01 (2016) 061 [arXiv:1509.03499] [INSPIRE]. 
[42] K. Dusling, M. Mace and R. Venugopalan, Multiparticle collectivity from initial state correlations in high energy proton-nucleus collisions, arXiv:1705.00745 [INSPIRE].

[43] K. Dusling, M. Mace and R. Venugopalan, Parton model description of multiparticle azimuthal correlations in pA collisions, arXiv:1706.06260 [INSPIRE].

[44] Y. Shi, C. Zhang and E. Wang, Multipole scattering amplitudes in the color glass condensate formalism, Phys. Rev. D 95 (2017) 116014 [arXiv:1704.00266] [INSPIRE].

[45] K. Fukushima and Y. Hidaka, Light projectile scattering off the color glass condensate, JHEP 06 (2007) 040 [arXiv: 0704 .2806] [INSPIRE].

[46] A. Dumitru and V. Skokov, Fluctuations of the gluon distribution from the small-x effective action, Phys. Rev. D 96 (2017) 056029 [arXiv: 1704.05917] [INSPIRE].

[47] H. Kowalski, L. Motyka and G. Watt, Exclusive diffractive processes at HERA within the dipole picture, Phys. Rev. D 74 (2006) 074016 [hep-ph/0606272] [INSPIRE].

[48] N. Borghini, P.M. Dinh and J.-Y. Ollitrault, Flow analysis from multiparticle azimuthal correlations, Phys. Rev. C 64 (2001) 054901 [nucl-th/0105040] [InSPIRE]. 